\title{
INEQUIDAD A PESAR DE LA REFORMA
}

\author{
Gloria Calvo* - Ariana Mina*
}

\section{RESUMEN}

La Reforma Educativa en Colombia, asociada con la expedición de la Ley General de Educación, ha tenido unos desarrollos diferentes a los de otros países Latinoamericanos. La descentralización pedagógica, por ejemplo, acompaña procesos de innovación en las prácticas pedagógicas de los docentes, amparada por la flexibilidad de los proyectos educativos institucionales. Al querer analizar los procesos de Reforma, los investigadores de El Aula Reformada se encontraron conque pese a los cambios introducidos por los procesos de reforma, la adquisición de la lectura y escritura no es equitativa y aprenden a leer y escribir mejor los alumnos $y$ alumnas de los colegios de élite.

\section{SUMMARY}

The colombian Educational reform - associated to the General Law of Education has evolved differently from other Latin American Countries. Pedagogical descentralization, for example, is conected to innovation processes in the pedagogical practices of the teachers, indorsed by institutional educational projects. When analyzing the reform process, the researchers of El Aula Reformada found that in spite of the changes introduced by the reform, the acquisition of reading and writing skills is not equitative, and that the students of the elite schools learn better to write and read than those from poor strata schools.

\section{Descriptores}

Políticas educacionales - Lectura - Escritura - Pedagogía - Didáctica

\section{Key words}

Education policies - Reading - Writing - Pedagogy - Didactics

La Reforma Educativa en Colombia se ha centrado en aspectos relacionados con la gestión escolar (Proyecto Educativo Institucional, Gobierno Escolar) y con la descentralización administrativa y financiera. Tal situación hace que la preocupación por los aprendizajes básicos (lectura, escritura y matemáticas) nazca desde los docentes comprometidos con estas asignaturas. Son ellos los que diseñan las

\footnotetext{
- Ponencia presentada en el Foro Internacional realizado en la Ciudad de Ensenada, Baja California, entre el 10 y 15 de Septiembre de 2001. Recoge algunos de los resultados de la investigación El aula Reformada en Colombia, estudio realizado bajo el convenio 230/00 firmado entre la Universidad Pedagógica Nacional y el Centro de Investigación y Acción Social -CIASde la Argentina, con financiación del Banco Interamericano de Desarrollo -BID-, dentro del Proyecto REDUC-BID II.

* Docente investigadora. Universidad Pedagógica Nacional. Coordinadora del proyecto REDUC - Colombia. Investigadora principal "El Aula Reformada”. E-mail: gcalvo@unipedagogica.edu.co.

* Bióloga. Magíster en Estudios Políticos. Co- investigadora "El Aula Reformada". Asistente de información de la Comisión Europea de Normalización CEN/ISS. E-mail: ariana_mina@hotmail.com. 
estrategias metodológicas innovadoras para un mayor logro en estas competencias y los que buscan integrarlas a la carta de navegación de la institución. La anterior posibilidad pone de presente la necesidad de que las reformas educativas se apuntalen desde abajo, desde los espacios de reflexión y de trabajo permanente en la escuela. Igualmente, llama la atención sobre la necesidad de sistematizar los productos de estas reflexiones que dan otro significado al currículo escolar y valorizan la cultura de los alumnos.

En Colombia, el proceso de descentralización educativa se ha traducido en un aumento en la cobertura de los servicios educativos, el incremento de la atención en las zonas rurales y en los sectores pobres, y en una apertura a los liderazgos y a los procesos de innovación ${ }^{95}$. De igual forma, se han evidenciado las posibilidades para mejorar la capacidad de gestión educativa y de construcción de un nuevo modelo social en las comunidades donde hay más firmeza en la decisión local y en donde se abren canales de participación y de comunicación para divulgar las experiencias ${ }^{96}$.

Pese a los grandes esfuerzos y a los avances generados a partir de la Reforma Educativa en Colombia, esta ponencia ilustra aspectos relacionados con la inequidad en la calidad de la educación, con el ánimo de evidenciar que todavía faltan procesos que brinden una educación de calidad a amplios sectores de la población menos favorecida social y culturalmente.

En los siguientes apartados se desarrollarán estas ideas.

\section{Civilizar el pueblo, formar las élites}

La historia de Colombia ha estado marcada por la exclusión de la mayoría de los colombianos del sistema político, lo cual se manifiesta para los propósitos de este estudio, en una clara diferenciación en la calidad de la educación que ofrecen las instituciones pertenecientes al sector privado y al sector oficial. El sistema político colombiano no ha reconocido, a lo largo de su trayectoria, la existencia del conjunto de su población. Esta exclusión se evidencia desde los aspectos normativos del Estado, como la Constitución, hasta los aspectos más básicos de la vida cotidiana, como la salud, la vivienda, la educación, la seguridad y la posibilidad de subsistencia económica. "En suma, la sociedad colombiana no ha conseguido realizar el ideal democrático de ofrecer educación de buena calidad a toda la población, ni el Estado cumplir uno de sus deberes básicos: el método de integrar la nación desde el aula" ${ }^{9}$. La Reforma educativa en Colombia no ha logrado un ideal de equidad y de justicia en tanto que no logra mitigar las contingencias naturales de los sectores menos favorecidos (Rawls, 1971). Siguen siendo los más favorecidos socialmente quienes acceden a una mejor educación y quienes cuentan con profesores con una inserción más completa en la modernidad. Esto genera inequidad en la calidad y en la efectividad de las estrategias pedagógicas y en última instancia, en la calidad de los resultados de la Prueba de Lenguaje REDUC ${ }^{\bullet}$ (ver cuadro No.1).

\footnotetext{
${ }^{95}$ CABALlERO, Piedad. Los hitos de la descentralización educativa en Colombia. Programa de Promoción de la Reforma Educativa en América Latina y el Caribe. PREAL- Instituto SER de Investigación, Julio-septiembre de 1999. Año 1, No. 2. También: Delgado, Adriana. "Reestructuración del Estado: reforma y modernización pendiente”. En: Revista Javeriana. No. 67. Agosto de 1999: 483-489.

${ }^{96}$ Banco Mundial -DNP-. La capacidad de los gobiernos locales: Mas allá de la asistencia técnica. Bogotá, 19 de julio de 1995.

${ }^{97}$ PALACIOS, Marco. Entre la legitimidad y la violencia: 1875-1994. Editorial Norma. Bogotá: 297.

- La prueba de lenguaje REDUC fue diseñada en México y aplicada en los países que participaron en la investigación El Aula Reformada (Chile, Colombia, Bolivia, El Salvador y once Estados de México). Estaba estructurada en dos partes. La primera, de 
Cuadro No. 1: Resultados por sector de la prueba de lenguaje REDUC aplicada a segundo grado de la básica primaria

\begin{tabular}{|l|c|l|}
\hline SECTOR & $\begin{array}{c}\text { INSTI } \\
\text { TUCIÓN }\end{array}$ & PUNTAJE \\
\hline Oficial & $\mathrm{O}_{1}$ & 7 \\
\hline & $\mathrm{O}_{2}$ & 8 \\
\hline & $0_{4}$ & 8 \\
\hline & $0_{3}$ & 9 \\
\hline \multicolumn{2}{|l|}{ Puntaje sector oficial } & 8 \\
\hline Privado & $\mathrm{P}_{1}$ & 9 \\
\hline \multicolumn{2}{|l|}{$\mathrm{P}_{2}$} & 10 \\
\hline \multicolumn{2}{|l|}{$\mathrm{P}_{3}$} & 13 \\
\hline \multicolumn{2}{|l|}{ Puntaje sector privado } & 15 \\
\hline \multicolumn{2}{|l|}{ Total } & 12 \\
\hline
\end{tabular}

Fuente: Elaboración propia a partir de los resultados cuantitativos obtenidos al corregir las pruebas de lenguaje REDUC (preguntas 1-19), siendo el máximo puntaje posible 19.

Las instituciones oficiales seleccionadas para el presente estudio, denominadas como $\mathrm{O}_{1} \mathrm{y}_{2}$, se encuentran ubicadas en sectores de desmarginalización urbana, aunque nominalmente $\mathrm{O}_{1}$ está clasificada como perteneciente al sector rural. $\mathrm{P}_{2}$ y $\mathrm{P}_{3}$, son de calendario $B$ y de clase media alta, mientras que $P_{1}$ es una institución privada

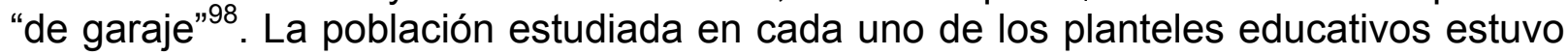
conformada por los alumnos de segundo grado de la básica primaria, y por los docentes encargados de las cátedras desarrolladas durante la hora de observación (profesoras de español). Las entrevistas realizadas a alumnos y alumnas siguieron una metodología de grupo focal ${ }^{99}$.

Los datos presentados en el cuadro anterior permiten concluir con Gajardo (1999) que aunque los procesos de reforma educativa han logrado avances importantes, los resultados todavía son magros. Pareciera que no existe una escuela pública de calidad para todos y que persisten las brechas entre estratos sociales; igualmente, los desniveles entre los conocimientos que adquieren los hijos de los grupos más pobres en la sociedad. Los sistemas escolares son altamente estratificados y no constituyen un mecanismo de movilidad social, con lo cual se evidencia que la reforma todavía no ha alcanzado sus objetivos de calidad, equidad y eficiencia ${ }^{100}$. Esta situación pone de presente una vez más la vigencia de la tesis de Helg, según la cual la historia de la educación en Colombia replica un mecanismo según el cual se civiliza el pueblo y se educan las élites. La investigadora suiza Aline Helg, al revisar las principales disposiciones legislativas sobre educación, colocando como eje temático la educación primaria y secundaria, concluye que en el pasado medio siglo, se institucionalizan dos

comprensión de lectura, tenía items para el uso de signos de puntuación; comprensión de información iconográfica y la segunda era un texto libre, a partir de la enunciación de un contexto.

${ }^{98}$ Término de uso común dentro del sector educativo para aludir a una educación privada de baja calidad.

${ }^{99}$ Loera, Armando. Los grupos de enfoque en la evaluación de los procesos de gerencia social. INDES. Octubre, 1999. Material mimeografiado.

${ }^{100}$ GAJARDO, M. 1999. Reformas educativas en América Latina. Balance de una década. PREAL, Documento No.15. Santiago, Chile. 50 páginas. 
redes educativas en las que el grado de conocimientos y adquisición de habilidades difiere ostensiblemente ${ }^{101}$. Tales hechos, ponen de manifiesto la debilidad del Estado central en el proceso de modernización educativa, ocasionada por la fragmentación regional y los intereses locales de las élites.

\section{Incorporación incompleta a la modernidad}

Un segundo elemento de análisis del Aula Reformada tiene relación con los docentes y su mayor o menor incorporación a la modernidad. Pese a que las credenciales académicas de las docentes estudiadas son equiparables, las condiciones en las cuales se lleva a cabo su quehacer pedagógico son diferentes. Si bien todas planean sus clases (tiempos, estrategias, actividades, evaluaciones), todas se inscriben dentro de corrientes pedagógicas innovadoras (constructivismo), las condiciones institucionales hacen la diferencia.

Cuadro No. 2: Trayectorias y actitudes de los Docentes

\begin{tabular}{|c|c|c|c|c|c|}
\hline Preguntas & 01 & $\mathrm{O} 2$ & $\mathrm{P} 1$ & P2 & P3 \\
\hline $\begin{array}{l}\text { 1. ¿Cuál es su máximo nivel } \\
\text { educativo? }\end{array}$ & Universidad & Universidad & Universidad & Universidad & Universidad \\
\hline $\begin{array}{l}\text { 2. ¿Cuántos años de } \\
\text { experiencia tiene como } \\
\text { docente? }\end{array}$ & 28 & 8 & 38 & 14 & 26 \\
\hline $\begin{array}{l}\text { 3. ¿Cuántos años lleva } \\
\text { enseñando en esta escuela? }\end{array}$ & 6 & 3 & 8 & 14 & 26 \\
\hline $\begin{array}{l}\text { 4. ¿Cómo fue asignado (a) a } \\
\text { esta escuela? }\end{array}$ & Traslado & Permuta & Traslado & Contratación & Contratación \\
\hline $\begin{array}{l}\text { 6. ¿Aparte de enseñar se } \\
\text { dedica a otras actividades } \\
\text { remuneradas? }\end{array}$ & No & No & No & No & No \\
\hline $\begin{array}{l}\text { 9. ¿En cuántas escuelas ha } \\
\text { enseñado en su carrera como } \\
\text { docente? }\end{array}$ & 6 & 3 & 16 & 0 & 0 \\
\hline \multicolumn{6}{|l|}{$\begin{array}{l}\text { 11. Indique el nivel de } \\
\text { satisfacción con los } \\
\text { siguientes aspectos de la } \\
\text { docencia }\end{array}$} \\
\hline a) Salario: & Algo S & Algo S & Algo $\mathrm{S}$ & Algo IS & Algo IS \\
\hline $\begin{array}{l}\text { b) Oportunidades de } \\
\text { desarrollo profesional: }\end{array}$ & Muy S & Muy S & Algo S & Algo S & Algo S \\
\hline c) Reconocimiento social: & Algo S & Algo S & Algo S & Algo S & Algo S \\
\hline d) Apoyo de los superiores: & Algo S & Algo S & Algo $\mathrm{S}$ & Algo $\mathrm{S}$ & Algo $S$ \\
\hline e) Ambiente en la escuela: & Algo S & Algo IS & Algo S & Muy S & Algo S \\
\hline f) Ambiente en la comunidad: & Algo S & Algo IS & Algo S & Muy S & Algo S \\
\hline $\begin{array}{l}\text { g) Influencia en las políticas } \\
\text { educativas en el país: }\end{array}$ & Algo IS & Algo IS & Algo S & Algo S & Algo S \\
\hline $\begin{array}{l}\text { h) Influencia en las } \\
\text { decisiones académicas: }\end{array}$ & Algo S & Algo S & Algo S & Algo S & Algo S \\
\hline $\begin{array}{l}\text { i) Influencia en las decisiones } \\
\text { administrativas en su } \\
\text { escuela: }\end{array}$ & Algo S & Muy S & Algo S & Algo S & Algo S \\
\hline $\begin{array}{l}\text { j) Control sobre su propia } \\
\text { aula: }\end{array}$ & Algo S & Algo S & Algo S & Muy S & Algo S \\
\hline
\end{tabular}

\footnotetext{
${ }^{101}$ HELG, Aline. 1987. La educación en Colombia. 1918-1957. Una Historia social, económica y política. Bogotá. Fondo Editorial Cerec. 334 páginas.
} 


\begin{tabular}{|l|l|l|l|l|l|}
\hline $\begin{array}{l}\text { n) Trabajo de equipo entre el } \\
\text { grupo de docentes: }\end{array}$ & Algo S & Muy IS & Algo S & Muy S & Algo S \\
\hline $\begin{array}{l}\text { ñ) Participación de los padres } \\
\text { de familia en asuntos de la } \\
\text { escuela: }\end{array}$ & Algo S & Muy IS & Algo S & Algo S & Algo S \\
\hline $\begin{array}{l}\text { o) Motivación de los } \\
\text { estudiantes en su propio } \\
\text { aprendizaje: }\end{array}$ & Algo S & Algo S & Algo S & Muy S & Algo S \\
\hline $\begin{array}{l}\text { p) Disciplina de los } \\
\text { estudiantes: }\end{array}$ & Algo S & Algo S & Algo S & Algo S & Algo S \\
\hline $\begin{array}{l}\text { 12. ¿Cuántos años más } \\
\text { considera que continuará en } \\
\text { el magisterio? }\end{array}$ & 10 & 25 & 0 & 9 & 8 \\
\hline
\end{tabular}

Leyenda:

Muy IS : muy insatisfactorio

Algo IS : algo insatisfactorio

Algo S : algo satisfactorio

Muy S: muy satisfactorio

Fuente : Elaboración realizada para la Investigación "El Aula Reformada" a partir de la información recopilada en los "cuestionarios para maestros". Bogotá - Colombia. 2000.

\section{Lo que nos dicen los cuestionarios analizados.}

- Reafirman la diferencia entre la educación privada de élite y la educación oficial. Llama la atención que ésta sea la evidencia que destacan los más recientes estudios que han sido difundidos en los últimos meses en el país. Con diferentes metodologías (basadas en los resultados del ICFES o en las pruebas CENSALES de la Secretaría de Educación del Distrito o con las pruebas aplicadas por el Laboratorio de la UNESCO), las investigaciones en cuestión remarcan esta inequidad ${ }^{102}$.

- Señalan el difícil papel del maestro. Rodrigo Parra y colaboradores (2000) han conceptualizado la situación de muchos maestros en Colombia bajo la categoría de Incorporación inconclusa. Primero, a la academia, que les otorga títulos pero no los incorpora a sus actividades; segundo, a la modernidad ya que pese a sus funciones relacionadas con la socialización de las nuevas generaciones, los códigos culturales de éstas les son casi extraños, dado el origen social de la mayoría de los docentes.

- La soledad del docente es mayor en el sector oficial: su trabajo es más aislado y es casi nula la confrontación con los pares.

- Los maestros enseñan a leer y a escribir con propuestas eclécticas, fruto de su práctica. Se generaliza aquello que fue para ellos exitoso.

- En las prácticas tradicionales prima el uso del tablero, de la copia, del coreado. Hay poco énfasis en el texto libre y pareciera que poco contara el interés del alumno.

- La institución $\mathrm{P}_{2}$ es consecuente en sus prácticas con su propuesta de Proyecto Pedagógico. También, aunque en menor grado, las maestras de la institución $\mathrm{P}_{4}$.

- Se puede corroborar la inferencia relacionada con la relativa ausencia de políticas sobre la lectura y la escritura. Las maestras conocen los lineamientos

\footnotetext{
${ }^{102}$ C.f. Dinero. "Los cincuenta mejores colegios". Abril 9 del 2001, No. 130, Pág. 30-44. También Dinero. "Los cien mejores colegios del 2000”. Abril del 2001, No. 131, Pág. 24-27.
} 
curriculares (sí a la pregunta No. 16), pero no saben si su manera de enseñar la lectura y escritura es compartida por otros docentes del país (pregunta No. 18).

- Por influencia de las corrientes constructivistas, con frecuencia el docente trata de definir su práctica desde esta teoría. Más en detalle, se constata que no se tienen en cuenta las experiencias del alumno, que sus intereses no inciden en la planificación de las actividades, que no se investiga.

- Las docentes encuestadas llevan más de quince años, en promedio, como maestras. Todas son post-graduadas.

- Una plaza docente es un trabajo estable.

- Pareciera que las docentes tienen la oportunidad de seleccionar el curso del nivel básico en el cual quieren enseñar.

- En las instituciones de mayor logro en la prueba de lenguaje REDUC existe mayor planeación de actividades. Allí las maestras asignan mas del $60 \%$ de su tiempo a enseñar, según la expresión utilizada al responder la pregunta No. 5.

Cuadro No. 3: Práctica docente

\begin{tabular}{|c|c|c|c|c|c|}
\hline Pregunta & $\mathrm{O}_{1}$ & $\mathrm{O}_{2}$ & $\mathrm{P}_{1}$ & $\mathrm{P}_{2}$ & $\mathrm{P}_{3}$ \\
\hline Facilita discusiones entre los estudiantes & $\begin{array}{l}\text { todos los } \\
\text { días }\end{array}$ & $\begin{array}{l}\text { todos los } \\
\text { días }\end{array}$ & $\begin{array}{l}\text { todos los } \\
\text { días }\end{array}$ & $\begin{array}{c}1-2 / \\
\text { semana }\end{array}$ & $\begin{array}{l}1-2 / \\
\text { seman } \\
a\end{array}$ \\
\hline Organiza a los estudiantes en equipos & $\begin{array}{l}\text { todos los } \\
\text { días }\end{array}$ & $\begin{array}{l}\text { todos los } \\
\text { días }\end{array}$ & $\begin{array}{l}\text { muy } \\
\text { pocas } \\
\text { veces }\end{array}$ & $\begin{array}{c}1-2 / \\
\text { semana }\end{array}$ & $\begin{array}{l}1-2 / \\
\text { seman } \\
\text { a }\end{array}$ \\
\hline Trabaja con estudiantes en forma personalizada & $\begin{array}{l}\text { todos los } \\
\text { días }\end{array}$ & $\begin{array}{l}\text { todos los } \\
\text { días }\end{array}$ & $\begin{array}{l}\text { todos los } \\
\text { días }\end{array}$ & $\begin{array}{c}1-2 / \\
\text { semana }\end{array}$ & $\begin{array}{l}1-2 / \\
\text { seman } \\
a\end{array}$ \\
\hline $\begin{array}{l}\text { Supervisa el trabajo de los estudiantes en sus } \\
\text { lugares }\end{array}$ & $\begin{array}{l}\text { todos los } \\
\text { días }\end{array}$ & $\begin{array}{l}\text { todos los } \\
\text { días }\end{array}$ & $\begin{array}{l}\text { todos los } \\
\text { días }\end{array}$ & $\begin{array}{l}\text { todos los } \\
\text { días }\end{array}$ & $\begin{array}{l}\text { todos } \\
\text { los días }\end{array}$ \\
\hline Leen lecturas seleccionadas por ellos & $\begin{array}{l}\text { todos los } \\
\text { días }\end{array}$ & $\begin{array}{l}1-2 / \\
\text { semana }\end{array}$ & \begin{tabular}{|l|}
$1-2$ \\
/semana
\end{tabular} & $\begin{array}{l}1-2 / \\
\text { mes }\end{array}$ & $\begin{array}{l}1-2 \\
\text { /mes }\end{array}$ \\
\hline Discute con otros estudiantes & $\begin{array}{l}\text { todos } \\
\text { los días }\end{array}$ & $\begin{array}{l}\text { todos los } \\
\text { días }\end{array}$ & $\begin{array}{l}\text { todos los } \\
\text { días }\end{array}$ & $\begin{array}{c}\text { Muy } \\
\text { pocas } \\
\text { veces }\end{array}$ & $\begin{array}{l}\text { Muy } \\
\text { pocas } \\
\text { veces } \\
\end{array}$ \\
\hline Comparte su trabajo con otros estudiantes & $\begin{array}{l}\text { todos } \\
\text { los días }\end{array}$ & $\begin{array}{l}1-2 / \\
\text { semana }\end{array}$ & $\begin{array}{l}\text { todos los } \\
\text { días }\end{array}$ & $\begin{array}{l}1-2 / \\
\text { mes }\end{array}$ & $\begin{array}{l}1-2 / \\
\text { mes }\end{array}$ \\
\hline $\begin{array}{l}\text { ¿Cuál es la estrategia para controlar la disciplina ue } \\
\text { usa de manera más frecuentemente? }\end{array}$ & $\begin{array}{l}\text { Amonestar } \\
\text { en privado }\end{array}$ & $\begin{array}{l}\text { Hablar con } \\
\text { el estudiante }\end{array}$ & $\begin{array}{l}\text { Interrogá } \\
\text { ndolos }\end{array}$ & $\begin{array}{r}\text { Dialogar } \\
\text { en } \\
\text { privado }\end{array}$ & $\begin{array}{l}\text { Amone } \\
\text { star en } \\
\text { privado }\end{array}$ \\
\hline
\end{tabular}

Fuente: Elaboración realizada para la investigación "El Aula Reformada" a partir de la información recopilada en los "cuestionarios para maestros". Bogotá - Colombia. 2000.

\section{EI PEI como expresión de cultura escolar}

La Ley General de Educación -Ley 115- fue enfática en exigir que cada plantel educativo debía contar en mayo de 1997 con un Proyecto Educativo Institucional que recogiera el sentir de la comunidad educativa y que se constituyera en su Carta de Navegación. 
De acuerdo con los postulados referentes a la socialización política de Almond (1996) y a los de Ávila y Camargo (1999), " por medio de la educación, el actor social incorpora estructuras de significación establecidas que orientan sus intercambios personales y lo vinculan, de múltiples maneras, a grupos, asociaciones, instituciones y organizaciones, por medio de significaciones compartidas expresadas en valores, normas y utopías sociales que le dan sentido a su acción dentro de la sociedad. La cultura se expresa así en los modos de organizar la vida para satisfacer las necesidades humanas, en las formas de pensar y concebir el mundo, en las maneras de vivir y valorar, y en las metas que comparten los miembros de un colectivo, pues todo ello orienta la acción individual y colectiva, permitiendo a los actores sociales una relación dinámica con la realidad social ${ }^{103}$.

Desde esta perspectiva, la cultura escolar es la matriz que permite la construcción de significaciones compartidas que le dan cohesión e identidad a la institución escolar, permitiendo el desarrollo de la pre-ciudadanía.

La Ley General de Educación, reconociendo la importancia de la institución escolar para la consolidación y reproducción de la democracia, buscó reivindicar su rol socializador, mediante herramientas como la construcción consensuada del Proyecto educativo Institucional - PEI - y la implementación de los Gobiernos Escolares. Pese a que teórica y normativamente, estos elementos potencian la transmisión de valores para la ciudadanía en las nuevas generaciones de colombianos, la ley ha encontrado numerosos obstáculos o aliados, según la institución educativa, en la cultura escolar. Es así como se observan casos en los cuales el PEI, el Manual de Convivencia y el Gobierno Escolar no son más que requisitos legales con los que se debe cumplir para tener la licencia de funcionamiento. Por el contrario, en otros planteles educativos, estas herramientas han iniciado procesos de construcción consensual, articulando mecanismos de socialización de la cultura política. Al respecto vale la pena señalar que a la luz de los resultados de la investigación de Ávila y Camargo (1999), se evidencia que las diferentes visiones que tienen sobre el PEI los miembros de la comunidad educativa, hacen que coexistan un PEI ideal -"entendido como un documento cuyo contenido recoge las ideas sobre el deber ser de la institución [...] plasma la imagen que la institución quiere mostrar o vender"104, con un PEI deseo - “ entendido como el conjunto de imágenes de deber ser, con las cuales el maestro otorga un sentido a su trabajo, sea que éstas las hayan derivado de sus procesos formativos o de su experiencia"105 - y con un PEI real -"entendido como aquel que de hecho funciona en la práctica, como resultado de todo lo que hacen los maestros por el simple hecho de trabajar en la escuela, aunque no expliciten sus intencionalidades, y aun cuando no estén construyendo la institución de una manera colectiva sino fragmentaria" ${ }^{106}$. De acuerdo con Velandia (1999), esta fragmentación de la cultura escolar constatada a través de la desarticulación de sus proyectos pone de presente que el $\mathrm{PEI}$, en tanto que proceso de construcción social de una visión compartida y de ejercicio de la democracia participativa, requiere de un mejoramiento continuo.

A la hora de evaluar los resultados de determinada política pública, se hace indispensable considerar los imaginarios que poseen los habitantes sobre los cuáles ésta incidió; la idea que tienen con respecto a cómo debería ser y funcionar la directriz política. Esta premisa se encuentra promovida en la actualidad, a través de las

\footnotetext{
${ }^{103}$ ÁVILA, R. Y CAMARGO, M. (1999). La Utopía de los PEI en el laberinto escolar. Bogotá, CIUP-COLCIENCIAS, PIIE, Página 104

${ }^{104}$ Idem, pág. 68.

105 Idem: 105.

106 Ibídem.

Digitalizado por RED ACADEMICA
} 
recomendaciones del INDES con respecto al diseño de políticas públicas. Se ha reevaluado la tradicional linealidad, para pasar a un sistema de concertación y priorización de las necesidades de las comunidades a la hora de trazar cualquier lineamiento político ${ }^{107}$. De esta manera, los ciudadanos tienen a estar de acuerdo en torno a los problemas propios de la sociedad y el gobierno gana un mayor grado de legitimidad. En esta misma línea filosófica en las políticas públicas, cobran especial importancia la retroalimentación, la evaluación sobre la marcha del desarrollo de los proyectos, tal como lo sugiere el modelo de formulación de políticas públicas de interacción -iteración y Peter Senge en la Quinta Disciplina ${ }^{108}$.

Sin embargo, esta cultura del consenso en el diseño, implementación y evaluación de las políticas públicas es aún lejano de la realidad colombiana, pese a que la Constitución de 1991 tiende a reconocer la pluralidad de intereses y la heterogeneidad de las condiciones de sus habitantes.

Por lo tanto, como lo señalan Ávila y Camargo (1999), “ en cuanto política, el PEI propone un cambio ideal, global, radical $\mathrm{y}$, en apariencia, rápido de la institución educativa. Está planeado para sacar a la organización escolar de un estado particular en que se encuentra, y dirigirla hacia un estado deseado, en general opuesto, sin pensar en las formas como ello podría hacerse, ni en los procesos de transición que deberían recorrerse, dada la heterogeneidad de las instituciones escolares en el país. Esta forma de entrega e implementación de la política es la que permite explicar el hecho de que, al inicio, los cambios son recibidos con un gran entusiasmo pero, con el tiempo, una cultura escolar, más arraigada de lo que parece, termina absorbiendo las iniciativas, y las innovaciones acaban por reacomodarse a las prácticas antiguas o tradicionales, cambiándolas de nombre y vaciando de contenido los esfuerzos de reforma. [...] Es más, en la estructura homogenizadora de la política, la cual desconoce la inmensa diversidad de la realidad a la que pretende cubrir, es donde podría ubicarse la razón fundamental de un posible fracaso. [...] Son, entonces, las exigencias con que se formula el PEI lo que lleva a constituirse en una política más, impuesta, sin posibilidad de apropiarse, pues se sobredimensiona el carácter prescriptivo superponiéndose a la misma realidad y conduciendo a una práctica mecánica que ignora las realizaciones, vivencias, conocimiento adquirido, historia de la institución y formación particular de los maestros" ${ }^{109}$.

A la luz de los supuestos teóricos planteados por Ávila y Camargo (1999) ${ }^{110}$, tanto la cultura escolar como la cultura contextual ${ }^{111}$ pueden funcionar como obstáculos o como elementos que potencien la implementación de una innovación, como la Reforma Educativa, introducida por la Ley General de Educación. Esto se logró ver a través de las inferencias realizadas para la presente investigación en las instituciones estudiadas, siendo las instituciones $\mathrm{P}_{2}$ y $\mathrm{P}_{3}$ casos en los cuales la cultura escolar genera una sinergia con la innovación, y $\mathrm{O}_{1}, \mathrm{O}_{2}$ y $\mathrm{P}_{1}$ casos en donde se dificulta la articulación de la Reforma.

Resulta ilustrativo en este sentido ver cómo pese a la vigencia de la normatividad y a su exigencia con respecto a la obligatoriedad de todo plantel educativo de contar

\footnotetext{
${ }^{107}$ MOLINA, Carlos Gerardo. Modelo interactivo de formación de politicas y programas sociales. Banco Interamericano de Desarrollo- Instituto Interamericano para el Desarrollo Social -INDES-. Washington.1996. 17 páginas.

${ }^{108}$ SENGE, Peter. La quinta disciplina. Editorial Barcena, Barcelona. 1992, 492 páginas.

${ }^{109}$ AVILA, R; CAMARGO, M. Op. cit. Página 58

${ }^{110}$ Idem

111 "Entendida como el conjunto de concepciones y prácticas cotidianas arraigadas en el ethos de los padres de familia y otros actores de la comunidad local y regional”. Ibídem. 
con un proyecto educativo institucional ${ }^{112}$, por ser concebidos como "una estrategia para el mejoramiento de la calidad de la educación que obedece a una política de auto-regulación" 113,3 de las 5 instituciones estudiadas afirmaron que no poseían aún dicho documento y $\mathrm{P}_{1}$ manifestó que el PEI era un documento privado al cual no se podía acceder. Llama la atención cómo frente a la situación de las anteriores instituciones mencionadas, $\mathrm{P}_{2}$ posee un PEI impreso, de libre circulación y distribución entre todos los miembros de la comunidad educativa y entre quienes sin pertenecer a ella, manifiesten su interés en conocerlo.

Las consideraciones anteriores, permiten afirmar la importancia de la cultura escolar en la implementación de las políticas, y en este caso en particular los procesos de reforma y a su vez, de la importancia de la cultura democrática en la elaboración, implementación, evaluación y reformulación de las políticas, tanto a nivel nacional, como entre los miembros de la comunidad hacia la cual van dirigidas las políticas; en este caso, la comunidad escolar. "En este marco el PEI se revela como un escenario privilegiado para el ejercicio de la democracia participativa que convoca a la reflexión, a la discusión y a los aportes sobre lo que se es y se quiere ser, como personas y como colectivo, partícipes de una comunidad educativa. La participación incluye no sólo la consulta acerca de la historia institucional, y el contexto y población a la cual sirve la institución, sino también la posibilidad de encauzar sus intereses y sus mejores potencialidades intelectuales y culturales, al servicio de la función socializadora que le ha sido encomendada" ${ }^{\prime 14}$.

\footnotetext{
${ }^{112}$ La Ley General de Educación (Ley 115 de 1994) dispone en su artículo 73: “Cada establecimiento educativo deberá elaborar y poner en práctica un Proyecto Educativo Institucional en el que se especifiquen, entre otros aspectos, los principios y fines del establecimiento, los recursos docentes y didácticos disponibles y necesarios, la estrategia pedagógica, el reglamento para docentes y estudiantes, y el sistema de gestión ...". Así mismo, uno de los decretos reglamentarios, expedido seis meses después de la Ley 115, el Decreto 1860 de Agosto 3 de 1994, establece la elaboración de Proyectos Educativos Institucionales como un mecanismo para expresar la forma como se han decidido alcanzar los fines de la educación en cada establecimiento, y para conseguir la autorregulación de las instituciones educativas. La exigencia llega hasta el señalamiento de "la fecha del 8 de febrero de 1997 como límite para que todos los establecimientos hayan adoptado y registrado en el Sistema Nacional de Información su proyecto Educativo Institucional" (Citado en AVILA, R; CAMARGO, M. Idem: 26)

113 ÁVILA, R; CAMARGO, M. Idem.

${ }^{114}$ Avila, R; Camargo, M. Op Cit: 95. 
Cuadro No. 4: Posibles situaciones institucionales frente a la implementación de la Reforma Educativa en Colombia

Las instituciones no han definido su PEl:

a. Ajustes

b. Falta de consenso

c. Realización a puerta cerrada, de manera vertical, expresando una tradición nacional en cuanto a la formulación de políticas públicas.

d. Inexistencia

e. Circulación restringida

EI PEI es la expresión de la filosofía Institucional

a. Apropiación

b. Consultable

c. Orientador de las estrategias pedagógicas

d. Dinamizador de las relaciones entre los distintos miembros de la comunidad educativa

e. Articulador y facilitador de la formación docente

Manual de Convivencia

a. Recoge las reglas de juego dentro de la comunidad educativa preservando la autonomía de los individuos.

b. Consigna prohibiciones y normas a las cuales los individuos se acogen de manera heterónoma.

Concepción de la evaluación como una estrategia pedagógica.

a. La evaluación es una estrategia de supervisión

b. La evaluación como forma de evidenciar logros y dificultades

Fuente : Elaboración realizada para el presente documento a partir de la información recopilada en la investigación "El Aula Reformada". Bogotá - Colombia. 2000

\section{Concepción de la evaluación como una estrategia pedagógica}

Los mayores o menores avances en la incorporación de la evaluación a los procesos de enseñanza-aprendizaje parecieran también estar relacionados con la equidad del sistema educativo. En este sentido, el análisis de las prácticas docentes, permitió observar dos maneras de entender la evaluación: la evaluación como una estrategia de supervisión o la evaluación como expresión de logros y dificultades. Estas dos maneras de concebir la evaluación reflejan una polarización en cuanto a la calidad de la educación que reciben unos y otros. En una sociedad en la cual no existe ya el empleo, potenciar la autonomía de los niños y las niñas es fundamental.

En las instituciones de mayor logro en la prueba de lenguaje REDUC (cfr. cuadro No. 1), se involucran estrategias de autoevaluación y de coevaluación (ver cuadro No. $5)$. 
Cuadro No. 5: Diálogo entre Docente y Estudiante en la institución P2

$P:$ ¿Y aquí qué paso?

A: Me puse a jugar...

$P$ : Te quedan 45 minutos y no has avanzado.

Eres tú quien maneja el tiempo. Mira a ver qué vas a hacer.

Fuente : Observación recopilada en el trabajo de campo realizado para "El aula Reformada”. Bogotá-Colombia, 2000.

\section{LAS ESTRATEGIAS PEDAGÓGICAS IMPLEMENTADAS EN EL AULA DE CLASE}

Los maestros en el aula despliegan una gran creatividad. Lamentablemente, creatividad no es sinónimo de calidad, ya que la multiplicidad de estrategias empleadas por los docentes en las instituciones estudiadas no se relacionaron con los puntajes más altos obtenidos en la Prueba de lenguaje REDUC. Pareciera que más que multiplicidad de estrategias es conveniente tener un propósito educativo definido, puede ser la filosofía del proyecto educativo institucional o una clara concepción pedagógica que sustente el proceso de enseñanza/aprendizaje. Así, en la institución codificada como P2 el centro de sus actividades lo constituye el trabajo por proyectos y asociadas al mismo, aparecen la creación de textos, el uso del diccionario y el trabajo por parejas. En este orden de ideas, el trabajo pedagógico por proyectos potencia habilidades para la convivencia, en la cual la interacción entre los alumnos(as) y entre éstos(as) y los(as) docentes genera un ambiente en donde se potencia tanto el trabajo individual como el cooperativo.

Las actividades desarrolladas en las clases observadas se presentan en el cuadro a continuación.

Cuadro No. 6: Inventario de las actividades analizadas mediante registro etnográfico

\begin{tabular}{|c|l|l|}
\hline INSTITUCION & \multicolumn{1}{|c|}{ ACTIVIDAD } & \multicolumn{1}{c|}{ FUENTE DE INFORMACION } \\
\hline $\mathrm{P}_{1}$ & Clase tradicional & $\begin{array}{l}\text { Registro basado en observación } \\
\text { participante. }\end{array}$ \\
\hline $\mathrm{P}_{2}$ & $\begin{array}{l}\text { Trabajo en parejas para un } \\
\text { proyecto. }\end{array}$ & $\begin{array}{l}\text { Vídeo } \\
\text { Entrevista a la maestra }\end{array}$ \\
\hline $\mathrm{P}_{3}$ & Concurso de lectura. & $\begin{array}{l}\text { Vídeo } \\
\text { Entrevista a la maestra }\end{array}$ \\
\hline $\mathrm{O}_{1}$ & $\begin{array}{l}\text { Taller sobre Educación } \\
\text { Sexual }\end{array}$ & $\begin{array}{l}\text { Vídeo } \\
\text { Registro basado en observación no } \\
\text { participante. } \\
\text { Entrevista a la maestra. }\end{array}$ \\
\hline $\mathrm{O}_{2}$ & Creación colectiva & $\begin{array}{l}\text { Vídeo } \\
\text { Registro basado en observación no } \\
\text { participante. } \\
\text { Entrevista a la maestra. }\end{array}$ \\
\hline
\end{tabular}

Fuente: Construido a partir de Información Primaria del Estudio. Bogotá, 2000

En un momento histórico en el cual la producción intelectual y el manejo oral y escrito de información en varios idiomas es absolutamente necesario para la 
competitividad del individuo, la adquisición de habilidades para el correcto manejo del lenguaje a través de la puntuación adquiere más relevancia que la copia del tablero o la separación de palabras por sílabas.

De igual forma, el uso creativo del diccionario sitúa al alumno en un contexto en el cual la información está disponible según sus intereses y necesidades, familiarizándolo con la realidad fuera del aula (ver cuadro 7).

Cuadro No. 7: Uso innovador del diccionario

- Profesora, ¿Cómo se escribe caza?

- Porqué no te remites al diccionario para que encuentres la palabra correcta.

- Porque la busqué y la definición que aparece, no me sirve.

El diccionario me define una casa!

- Ah! ¿Qué otras posibilidades tienes para escribir la palabra?

- Escribirla con z! Voy a mirar a ver si esa es!

- Búscala haber si esa definición si te sirve.

Fuente: Videograbación de una clase de español en la Institución $P_{2}$. Bogotá, 2000.

A la luz de los resultados del presente estudio, una posible orientación pedagógica, podría contemplar, por lo menos cuatro aspectos:

- El trabajo por proyectos.

- La coordinación y la posibilidad de colaboración entre los distintos miembros de la comunidad educativa (capital social).

- La filosofía institucional plasmada en las distintas dimensiones del proyecto pedagógico institucional.

- La explicitación del propósito formativo de la estrategia pedagógica seleccionada.

Estos elementos permiten la conformación de matriz cultural, en donde el consenso potencia la conformación de una visión común en lo educativo y en lo pedagógico.

A manera de síntesis, el cuadro No. 8 presenta las principales formas de enseñar teniendo en cuenta si la práctica pedagógica puede ser clasificada como tradicional o como innovadora. 
Cuadro No. 8: Características de las prácticas docentes

\begin{tabular}{|c|c|c|}
\hline Características & Tradicional & Innovadora \\
\hline Escritura & $\begin{array}{l}\text { - Copia del tablero, del } \\
\text { libro o del periódico. }\end{array}$ & $\begin{array}{l}\text { - Énfasis en los textos } \\
\text { libres. }\end{array}$ \\
\hline Gramática & $\begin{array}{l}\text { • División de palabras } \\
\text { por sílabas. }\end{array}$ & $\begin{array}{l}\text { • Énfasis en el uso de la } \\
\text { puntuación. }\end{array}$ \\
\hline Intencionalidad & $\begin{array}{l}\text { - Se privilegia la } \\
\text { memoria. }\end{array}$ & $\begin{array}{l}\text { - Fomento de la } \\
\text { autonomía }\end{array}$ \\
\hline Diccionario & $\begin{array}{l}\text { - Se usa el diccionario } \\
\text { para copiar definiciones. }\end{array}$ & $\begin{array}{l}\bullet \quad \text { Uso creativo del } \\
\text { diccionario }\end{array}$ \\
\hline Evaluación & $\begin{array}{l}\text { - La evaluación es una } \\
\text { estrategia de supervisión. }\end{array}$ & $\begin{array}{l}\text { • Evaluación como forma } \\
\text { de evidenciar logros y } \\
\text { dificultades }\end{array}$ \\
\hline Metodología & $\begin{array}{l}\text { - Ausencia de } \\
\text { proyecto Pedagógico. }\end{array}$ & $\begin{array}{l}\text { - Trabajo pedagógico por } \\
\text { proyectos }\end{array}$ \\
\hline Materiales & $\begin{array}{l}\text { - Ausencia de textos } \\
\text { - Acceso restringido a } \\
\text { los materiales educativos }\end{array}$ & $\begin{array}{l}\text { - Uso creativo de los } \\
\text { libros de texto. } \\
\text { - Centros de recursos } \\
\text { con acceso libre. }\end{array}$ \\
\hline $\begin{array}{l}\text { Elaboración del } \\
\text { PEI }\end{array}$ & $\begin{array}{l}\text { • PEI elaborado por } \\
\text { los directivos }\end{array}$ & - PEI consensuado. \\
\hline
\end{tabular}

Fuente: Análisis de portafolios y de la videograbación de la práctica del docente. Bogotá, 2000.

A manera de síntesis, el cuadro No. 9 permite ver la inequidad en las prácticas en las instituciones analizadas.

Cuadro No.9: Características de la práctica docente por institución

\begin{tabular}{|c|c|c|c|c|}
\hline Institución & $\begin{array}{c}\text { Finalidad de } \\
\text { las prácticas }\end{array}$ & $\begin{array}{c}\text { Manejo de } \\
\text { estrategias }\end{array}$ & $\begin{array}{c}\text { Nivel de } \\
\text { generalización }\end{array}$ & $\begin{array}{c}\text { Nivel de } \\
\text { Logro } \\
\text { Ineficaz }\end{array}$ \\
\hline O1 & No sistemática & Creativa & Propia & Ineficaz \\
\hline O2 & No sistemática & Creativa & Común & Ineficaz \\
\hline O3 & No sistemática & Tradicional & Común & Ineficaz \\
\hline O4 & Sistemática & Tradicional & Común & Ineficaz \\
\hline P1 & Anárquica & Creativa & Propia & Eficaz \\
\hline P2 & Sistemática & Innovadora & Propia & Eficaz \\
\hline P3 & Sistemática & Innovadora & Propia & Eficaz \\
\hline P4 & No sistemática & Creativa & Propia & \\
\hline
\end{tabular}

Fuente: Construido a partir de Información Primaria del Estudio. Bogotá, 2000

En este orden de ideas, el Trabajo pedagógico por proyectos potencia habilidades para la convivencia, en la cual la interacción entre los alumnos(as) y entre éstos(as) y los(as) docentes genera un ambiente en donde se potencia tanto el trabajo individual como el cooperativo. 


\section{LOS RESULTADOS DE LA PRUEBA DE LENGUAJE REDUC}

Finalmente, vale la pena mostrar cómo los resultados de la Prueba de Lenguaje REDUC también permiten ver la inequidad de los aprendizajes entre el sector oficial y el sector privado (ver cuadro No. 10).

Cuadro No. 10: Ubicación de las instituciones estudiadas según el puntaje obtenido en cada uno de los tipos de ejercicios de la prueba de lenguaje REDUC

\begin{tabular}{|l|c|c|c|c|c|c|c|c|}
\hline \multicolumn{2}{|l|}{ Posicionamiento de las instituciones estudiadas según el puntaje obtenido } \\
\hline Tipo de ejercicio & 1 & 2 & 3 & 4 & 5 & 6 & 7 & 8 \\
\hline \multirow{2}{*}{ Comprensión Lectora } & $\mathrm{O}_{1}$ & $\mathrm{P}_{4}$ & $\mathrm{O}_{4}$ & $\mathrm{O}_{3}$ & $\mathrm{O}_{2}$ & $\mathrm{P}_{1}$ & $\mathrm{P}_{3}$ & $\mathrm{P}_{2}$ \\
& $(1.45 / 3)$ & $(2 / 3)$ & $2.14 / 3)$ & $(2.3 / 3)$ & $(2.55 / 3)$ & $(2.6 / 3)$ & $(2.94 / 3)$ & $2.95 / 3)$ \\
\hline \multirow{2}{*}{ Epístola } & $\mathrm{O}_{1}$ & $\mathrm{O}_{2}$ & $\mathrm{O}_{4}$ & $\mathrm{O}_{3}$ & $\mathrm{P}_{1}$ & $\mathrm{P}_{4}$ & $\mathrm{P}_{3}$ & $\mathrm{P}_{2}$ \\
& $(0.3 / 2)$ & $(0.45 / 2)$ & $(0.49 / 2)$ & $(0.5 / 2)$ & $(0.6 / 2)$ & $(0.7 / 2)$ & $(1.1 / 2)$ & $(1.2 / 2)$ \\
\hline \multirow{2}{*}{ Gramática } & $\mathrm{O}_{2}$ & $\mathrm{O}_{3}$ & $\mathrm{O}_{1}$ & $\mathrm{P}_{1}$ & $\mathrm{O}_{4}$ & $\mathrm{P}_{4}$ & $\mathrm{P}_{3}$ & $\mathrm{P}_{2}$ \\
& $(3.65 / 11)$ & $(3.95 / 11)$ & $(4.3 / 11)$ & $(4.3 / 11)$ & $(4.63 / 11)$ & $(5.3 / 11)$ & $(7.2 / 11)$ & $(8.4 / 11)$ \\
\hline \multirow{2}{*}{ Información iconográfica } & $\mathrm{O}_{1}$ & $\mathrm{O}_{4}$ & $\mathrm{O}_{2}$ & $\mathrm{P}_{1}$ & $\mathrm{P}_{4}$ & $\mathrm{O}_{3}$ & $\mathrm{P}_{3}$ & $\mathrm{P}_{2}$ \\
& $(1.35 / 3)$ & $(1.45 / 3)$ & $(1.6 / 3)$ & $(2 / 3)$ & $(2 / 3)$ & $(2.1 / 3)$ & $(2.3 / 3)$ & $(2.5 / 3)$ \\
\hline
\end{tabular}

Fuente: Resultados de las Pruebas de lenguaje REDUC aplicadas al segundo grado de la básica primaria en ocho instituciones educativas colombianas. Cuadro elaborado para el presente informe.

La presentación de los resultados de la Prueba de lenguaje REDUC discriminada por el tipo de ejercicio, permite evidenciar cómo las instituciones privadas de élite siguen obteniendo los mejores puntajes tal como ya había sido señalado en el cuadro No. 1. Siguiendo con la tesis abordada en el presente artículo, el cuadro No. 11 evidencia cómo los niveles más bajos en la estructuración de textos libres se encuentra entre los estudiantes de los planteles oficiales.

Cuadro No. 11: Resultados por sector de la prueba de lenguaje REDUC aplicada a segundo grado de la básica primaria

\begin{tabular}{|c|c|c|c|c|}
\hline \multirow{2}{*}{ INSTITUCIÓN } & \multicolumn{4}{|c|}{ Calificación del texto libre } \\
\cline { 2 - 5 } EDUCATIVA & $\mathrm{A}$ & $\mathrm{B}$ & $\mathrm{C}$ & $\mathrm{D}$ \\
\hline $\mathrm{O}_{1}$ & $50 \%$ & $37.5 \%$ & $12.5 \%$ & $0 \%$ \\
\hline $\mathrm{O}_{2}$ & $50 \%$ & $38 \%$ & $4 \%$ & $8 \%$ \\
\hline $\mathrm{O}_{3}$ & $15 \%$ & $11 \%$ & $7 \%$ & $0 \%$ \\
\hline $\mathrm{O}_{4}$ & $50.8 \%$ & $39.2 \%$ & $7.84 \%$ & $1.9 \%$ \\
\hline $\mathrm{P}_{1}$ & $80 \%$ & $10 \%$ & $10 \%$ & $0 \%$ \\
\hline $\mathrm{P}_{2}$ & $9 \%$ & $17 \%$ & $22 \%$ & $52 \%$ \\
\hline $\mathrm{P}_{3}$ & $52 \%$ & $11 \%$ & $7 \%$ & $0 \%$ \\
\hline $\mathrm{P}_{4}$ & $48.3 \%$ & $31 \%$ & $10.3 \%$ & $10.3 \%$ \\
\hline
\end{tabular}

Fuente: elaboración propia a partir de los resultados cualitativos obtenidos al corregir las pruebas de lenguaje REDUC (texto libre) en cada una de las instituciones estudiadas.

Los mejores resultados de la Prueba de Lenguaje REDUC parecieran estar relacionados con una mayor apropiación de las grandes directrices de la Reforma Educativa en Colombia. Así, la institución $\mathrm{P}_{2}$, que es la que obtiene los mejores 
resultados, cuenta con un proyecto educativo institucional ampliamente conocido y apropiado por la comunidad educativa; igualmente, trabaja con modalidades de autoevaluación y los problemas se manejan de acuerdo con los postulados de la "negociación de conflictos" de la escuela de Harvard. Además, la formación de los profesores corresponde a planes institucionales y las estrategias didácticas se centralizan alrededor del trabajo por proyectos. En síntesis, en esta institución confluye articuladamente los diferentes componentes de la Reforma Educativa colombiana. Esta también parece ser la situación presente en la institución $\mathrm{P}_{3}$, filial de un colegio europeo.

En el polo opuesto, se hallan las instituciones oficiales analizadas en el presente estudio. En ellas, el proyecto educativo institucional está en ciernes, el manual de convivencia no supera la normatividad estipulada por las directivas, la evaluación se realiza de manera heterónoma y las estrategias pedagógicas no tienen un propósito definido. Esta realidad podría explicar los bajos resultados obtenidos por estas instituciones en la Prueba de lenguaje REDUC y pone de presente la diferencia entre la educación privada de élite y la oficial.

A medio camino entre la educación oficial y la privada de élite se encuentran las instituciones $\mathrm{P}_{1}$ y $\mathrm{P}_{4}$. La primera, pese a tener las condiciones económicas para ofrecer una educación de calidad, expresa muchos de los problemas socio culturales que caracterizan la clase emergente colombiana. La segunda, de naturaleza mixta, expresa algunas bondades de la política educativa, como la presencia de docentes subsidiados por el Estado, bonificaciones por el número de alumnos, merienda escolar y la participación en proyectos institucionales. No obstante, la política educativa no mitiga a cabalidad las contingencias naturales de sus estudiantes.

\section{CONSIDERACIONES FINALES}

- Los resultados del Aula Reformada en Colombia, evidencian que la incorporación e implementación de la Reforma en las directrices anteriores, se ha dado de manera desigual en las instituciones escolares. Las instituciones que tienen una tradición de innovación previa a la expedición de la Ley 115, han hecho una mejor apropiación de la Reforma. En estos casos, la normativa no fue más que la regulación de un proceso que ya venía en curso; en tanto que para otras instituciones, quizá más tradicionales o menos comprometidas con su labor, la norma fue vista como un requisito que se debía cumplir - con o sin consenso-, o recogiendo la idiosincrasia del país, evadir.

- La heterogeneidad de los resultados permite evidenciar la persistencia de inequidades en la educación (ver cuadro No. 4.2: Resultados por sector de la prueba de lenguaje REDUC aplicada a segundo grado de la básica primaria). Mientras el puntaje obtenido por los colegios oficiales es de 8/19, el de los privados es de 12/19. Sin embargo, los resultados globales siguen siendo deficientes (10/19), situación que corrobora los resultados obtenidos por Colombia en el TIMMSS, prueba en la cual obtuvo el puesto 40 entre 41 países examinados, "es decir, un lugar por debajo de todos los países de Asia, Europa Oriental y Oriente Medio, que participaron en el estudio" 115 .

\footnotetext{
${ }^{115}$ WOLFF, L. 1997. Las evaluaciones educacionales en América Latina. Avance actual y futuros desafios. PREAL/ Documentos, Santiago, Chile. 
- Aún a riesgo de ser reiterativas, hay que traer a colación la reflexión de Marcela Gajardo que se proponía al inicio de este texto. Según esta autora pareciera que no existe una escuela pública de calidad para todos y que persisten las brechas entre estratos sociales; igualmente, los desniveles entre los conocimientos que adquieren los hijos de los grupos más pobres en la sociedad. Los sistemas escolares son altamente estratificados y no constituyen un mecanismo de movilidad social, con lo cual se evidencia que la reforma todavía no ha alcanzado sus objetivos de calidad, equidad y eficiencia ${ }^{116}$.

${ }^{116}$ GAJARDO, M. 1999. Reformas educativas en América Latina. Balance de una década. PREAL, Documento No.15. Santiago, Chile. 50 páginas. 